Weimarer Nietzsche-Bibliographie 
Personalbibliographien zur neueren deutschen Literatur, herausgegeben von Michael Knoche und Reinhart Tgahrt. Band 4/1 
Stiftung Weimarer Klassik

Herzogin Anna Amalia Bibliothek

\section{Weimarer Nietzsche-Bibliographie (WNB)}

Bearbeitet von

Susanne Jung, Frank Simon-Ritz, Clemens Wahle, Erdmann von Wilamowitz-Moellendorff, Wolfram Wojtecki

Band 1: Primärliteratur 1867-1998

Verlag J. B. Metzler

Stuttgart · Weimar 
Projektleitung: Michael Knoche

Erarbeitet mit Unterstützung der Deutschen Forschungsgemeinschaft

Die Deutsche Bibliothek - CIP-Einheitsaufnahme

Weimarer Nietzsche-Bibliographie : (WNB) / Stiftung Weimarer Klassik, Herzogin-AnnaAmalia-Bibliothek. Bearb. von Susanne Jung ... - Stuttgart ; Weimar : Metzler

(Personalbibliographien zur neueren deutschen Literatur ; Bd. 4)

ISBN 978-3-476-01651-5

Bd. 1. Primärliteratur 1867 - 1998. - 2000

ISBN 978-3-476-01646-1

ISBN 978-3-476-01651-5 (Gesamtwerk)

ISBN 978-3-476-01646-1

ISBN 978-3-476-02730-6 (eBook)

DOI 10.1007/978-3-476-02730-6

Dieses Werk einschließlich aller seiner Teile ist urheberrechtlich geschützt. Jede Verwertung außerhalb der engen Grenzen des Urheberrechtsgesetzes ist ohne Zustimmung des Verlages unzulässig und strafbar. Das gilt insbesondere für Vervielfältigungen, Übersetzungen, Mikroverfilmungen und die Einspeicherung und Verarbeitung in elektronischen Systemen.

(C) 2000 Springer-Verlag GmbH Deutschland

Ursprünglich erschienen bei J.B. Metzlersche Verlagsbuchhandlung

und Carl Ernst Poeschel Verlag GmbH in Stuttgart 2000

www.metzlerverlag.de

info@metzlerverlag.de 


\section{Vorwort}

»Jeden Schriftsteller überrascht es von neuem, " heißt es in >Menschliches, Allzumenschliches`, "wie das Buch, sobald es sich von ihm gelöst hat, ein eigenes Leben für sich weiterlebt; es ist ihm zumute, als wäre der eine Teil eines Insektes losgetrennt und ginge nun seinen eigenen Weg weiter. Vielleicht vergißt er es fast ganz, vielleicht erhebt er sich über die darin niedergelegten Ansichten, vielleicht selbst versteht er es nicht mehr und hat jene Schwingen verloren, auf denen er damals flog, als er jenes Buch aussann: währenddem sucht es sich seine Leser, entzündet Leben, beglückt, erschreckt, erzeugt neue Werke, wird die Seele von Vorsätzen und Handlungen - kurz: es lebt wie ein mit Geist und Seele ausgestattetes Wesen und ist doch kein Mensch. ${ }^{1}$

Bei keinem Schriftsteller dürfte die Überraschung über das Eigenleben seiner Werke größer gewesen sein als bei Nietzsche selbst. Sein Philosophieren jenseits der großen Systeme des 19. Jahrhunderts bot und bietet Gelegenheit für höchst unterschiedliche Anknüpfungspunkte in Wissenschaft, Kunst und Politik. Die Breite der Rezeption ist genauso außergewöhnlich wie ihre Widersprüchlichkeit. Sein Werk hat im 20. Jahrhundert Leser weit über Deutschland hinaus gefunden und eine Sprengkraft wie das keines anderen Denkers entfaltet. Am 25. August 1900 ist Friedrich Nietzsche in Weimar gestorben. Einhundert Jahre nach seinem Tod ist es an der Zeit, Leben, Werk und Wirkung Nietzsches mit den nüchternen Mitteln der Bibliographie zu bilanzieren.

Im vorliegenden Band zur Primärliteratur werden die Werke von ihren Erstausgaben über die wiederholten Neuausgaben einzelner Bücher samt ihrer Auflagen bis hin zu den Werkausgaben sowie in analoger Weise Übersetzungen von Werken Nietzsches in 42 Sprachen nachgewiesen. Die übrigen vier Bände, die im Jahr 2001 folgen, dokumentieren systematisch geordnet - die Sekundärliteratur zu Leben, Werk und Wirkung. Die Berichtszeit der Bibliographie erstreckt sich von 1867, dem Jahr der ersten Publikation eines Textes durch Nietzsche, bis zum Jahr 1998, d. h. über einen Zeitraum von 131 Jahren.

$\mathrm{Da}$ sich die Herzogin Anna Amalia Bibliothek dieser Aufgabe angenommen hat, ist in erster Linie in ihrer Nietzsche-Sammlung begründet, die aus drei Teilen besteht: dem privaten Büchernachlaß Nietzsches, den Beständen des ehemaligen Nietzsche-Archivs und einer ergänzenden Sammlung an Nietzsche-Literatur. Elisabeth Förster-Nietzsche hatte die Bücher ihres Bruders, die nach seinem Zusammenbruch in Turin an verschiedenen Aufbewahrungsorten aufzufinden waren, zusammengetragen und seit 1896 im Weimarer Nietzsche-Archiv aufbewahrt. 1946 sind sie von dort zunächst an das Goethe- und Schiller-Archiv gelangt und 1954 an die neugegründete Zentralbibliothek der deutschen Klassik unter dem Dach der Nationalen Forschungs- und Gedenkstätten der klassischen deutschen Literatur in Weimar übergegangen. Jetzt gehören

${ }^{1}$ Menschliches, Allzumenschliches, 1. Band. SA 1, 569f. Für die Auflösung der Abkürzungen siehe S. XI-XIV. 
sie als Sondersammlung zur Herzogin Anna Amalia Bibliothek. Den besonderen Quellenwert dieses Bestandes machen zahlreiche Lesespuren und Marginalien Nietzsches in jenen Büchern aus, denen sein spezifisches Interesse galt.

Der zweite Teil der Nietzsche-Sammlung der Herzogin Anna Amalia Bibliothek umfaßt die Bibliothek des ehemaligen Nietzsche-Archivs. Es handelt sich im wesentlichen um eine Sammlung von Literatur über Nietzsche sowie um Drucke seiner Werke von den Erstdrucken bis 1945. Außerdem sind Teile der Privatbibliothek von Elisabeth FörsterNietzsche mit zahlreichen Widmungsexemplaren von Zeitgenossen enthalten. Den dritten Teil der Nietzsche-Sammlung bildet die vor allem nach 1955 erschienene Primär- und Sekundärliteratur, darunter auch einige Übersetzungen. Erst seit der Wende 1989/90 ist die Herzogin Anna Amalia Bibliothek in der Lage, diesen Bestand zielgerichtet auszubauen. Nietzsches Manuskripte und Briefe sowie die Unterlagen des NietzscheArchivs befinden sich im Goethe- und Schiller-Archiv, das wie die Herzogin Anna Amalia Bibliothek zur Stiftung Weimarer Klassik gehört.

Damit verfügt Weimar über eine geeignete Materialbasis für ein bibliographisches Unternehmen dieser Art. Es besteht aber auch eine Art Pflicht zur Wiedergutmachung. Denn zu DDR-Zeiten war die NietzscheSammlung der Zentralbibliothek der deutschen Klassik im wesentlichen nur einem kleinen Kreis internationaler Nietzsche-Forscher zugänglich, den Normalbenutzern aber blieben seine Texte vorenthalten - aus ideologischen, nicht aus konservatorischen Gründen. Auf den Katalogkärtchen ist noch heute der Stempelvermerk "Nachweis des besonderen Benutzungszwecks erforderlich« erkennbar. Der Nietzsche-Bestand ist über die Zeiten gerettet worden, aber er war offiziell kein Thema und tatsächlich keiner besonderen Anstrengung wert.

Schließlich sprach für die Ansiedlung der Nietzsche-Bibliographie an der Herzogin Anna Amalia Bibliothek auch der methodische Erfahrungsschatz der Weimarer Bibliothekare. Die bibliographische Arbeit hat hier eine vierzigjährige Tradition und bildet heute einen Eckstein im Konzept Forschungsbibliothek. ${ }^{2}$ Seit 1960 wird jährlich die »Internationale Bibliographie zur deutschen Klassik" bearbeitet und seit einigen Jahren über den Münchener Saur-Verlag veröffentlicht. Hinzu kommen seit vielen Jahren die regelmäßig aktualisierten retrospektiven Personalbibliographien zu Lessing, Wieland, Herder, Goethe, Schiller und Heine. Auch ein methodisch ganz andersartiges und in vieler Hinsicht neues Projekt gehört zum Arbeitsprogramm: die analytische Erschließung des Weimarer »Journal des Luxus und der Moden« aus dem Verlag von Friedrich Justin Bertuch.

${ }^{2}$ Michael Knoche: Die Forschungsbibliothek. Umrisse eines in Deutschland neuen Bibliothekstyps. In: Bibliothek - Forschung und Praxis 17 (1993), S. 291300. 


\section{Entstehung des Projekts und Aufgabenverteilung}

Eine umfassende Bibliographie ist trotz vieler vorhandener Ansätze immer noch ein Desiderat der Nietzsche-Forschung. Die Schwierigkeit besteht im Umfang und in der Komplexität der internationalen Ausstrahlung Nietzsches auf die verschiedensten Wissenschaften wie Philosophie, Germanistik, klassische Philologie, Kunstwissenschaft, Musikwissenschaft, Theologie, Religionswissenschaft und Psychologie. Wolfgang Müller-Lauter war einer derjenigen, die immer wieder - auch in persönlichen Gesprächen 1993/94 in Weimar - auf die Notwendigkeit einer bibliographischen Dokumentation hingewiesen haben. Mazzino Montinari hatte schon vor Jahren von einer "schmerzlichst empfundene(n) Lücke « gesprochen. ${ }^{3}$ Unter den Bibliothekaren der Herzogin Anna Amalia Bibliothek war es Erdmann von Wilamowitz-Moellendorff, der sich das Projekt zueigen gemacht und für die Verwirklichung besonders eingesetzt hat (Projektvorbereitung, Entwurf der Systematik). ${ }^{4}$ Im Herbst 1995 waren nach der Förderungszusage durch die Deutsche Forschungsgemeinschaft die finanziellen, organisatorischen und personellen Voraussetzungen für das Projekt geschaffen. Die beiden DiplomBibliothekare Susanne Jung und Clemens Wahle sowie die wissenschaftlichen Mitarbeiter Dr. Frank Simon-Ritz (ab 1999 Dr. Wolfram Wojtecki) und Erdmann von Wilamowitz-Moellendorff nahmen ihre Arbeit auf. Die Arbeitsgruppe wird bis zum Sommer 2001 fortbestehen.

Bis zu seinem Ausscheiden im Herbst 1999 hatte Frank Simon-Ritz die Funktion eines Projektkoordinators inne und führte das Projekt über zahlreiche, insbesondere über Klippen der EDV-Entwicklung. Er ergriff auch die Initiative, die im Entstehen begriffene Datenbank möglichst frühzeitig ausgewählten Nietzsche-Forschern über das Internet zugänglich zu machen und auf diese Weise in einen ständigen Austausch mit der Forschung einzutreten. Diese Datenbank wiederum war Ausgangspunkt eines DFG-Kolloquiums mit verallgemeinerter Fragestellung (»Bibliographische Datenbanken im Internet « vom 4. bis 5. Dezember 1997 an der Herzogin Anna Amalia Bibliothek), auf dem Verleger, Bibliographen, Bibliothekare und Wissenschaftler über künftige Publikationsformen von bibliographischen Daten miteinander ins Gespräch kamen. ${ }^{5}$ Erdmann von Wilamowitz-Moellendorff hat als Projektkoordinator ab 1999 vor allem die Aufgabe, die Drucklegung in zwei Etappen termingerecht sicherzustellen.

3 Zitiert bei Wolfgang Müller-Lauter: Zwischenbilanz. Zur Weiterführung der von Montinari mitbegründeten Nietzsche-Editionen nach 1986. In: NietzscheStudien 23 (1994), S. 310.

${ }^{4} \mathrm{Vgl}$. auch Erdmann von Wilamowitz-Moellendorff: Die bibliographische Erschließung von Leben, Werk und Wirkung Friedrich Nietzsches - ein Bericht zur Lage, mit einem Ausblick auf das Projekt `Nietzsche-Bibliographie` der Herzogin Anna Amalia Bibliothek Weimar. In: Nietzsche-Studien 26 (1997), S. 551-573.

${ }^{5}$ Die Vorträge können über die Internet-Homepage der Stiftung Weimarer Klassik / Herzogin Anna Amalia Bibliothek abgerufen werden: www.weimarklassik.de/haab/index/html. 
Der Hauptanteil der Erarbeitung und Redaktion des vorliegenden Bandes »Primärliteratur«, d.h. die Ermittlung, formale und inhaltliche Erschließung der in ihm enthaltenen Titel, lag in den Händen von Clemens Wahle. Er hatte dabei unter anderem die schwierige Aufgabe zu bewältigen, die Gedichtsammlungen aufzuschlüsseln und einzelne Gedichte oder Textauszüge im Gesamtwerk zu verorten. Doch ist die Weimarer Nietzsche-Bibliographie mehr als jedes vergleichbare Projekt Resultat der Arbeit eines Teams. Alle wichtigen Entscheidungen, zu denen auch die Leiterin der Bibliographischen Abteilung der Herzogin Anna Amalia Bibliothek, Doris Kuhles, beigetragen hat, sind in der Arbeitsgruppe gemeinsam getroffen worden. Die strikt alphabetische Auflistung der Namen auf dem Titelblatt soll diese Gemeinschaftsleistung unterstreichen.

Von Anfang an war die Weimarer Nietzsche-Bibliographie so konzipiert $^{6}$, daß sie auch in einer Online-Version verfügbar sein sollte. Im Winter 1997/98 konnte eine erste Fassung dieser Datenbank einem Kreis von Korrespondenten und interessierten Nietzsche-Forschern zugänglich gemacht werden; sie stieß auf ein breites Interesse. Eine aus den bisherigen Erfahrungen resultierende Weiterentwicklung des Online-Zuganges zur Datenbank wird ins Internet gestellt werden, wobei Zeitpunkt und Konditionen noch offen sind. Auch eine Weiterführung der Bibliographie über 1998 wird angestrebt. Die Realisierung hängt allerdings davon ab, ob für diese Aufgabe personelle Ressourcen auf Dauer erschlossen werden können.

\section{Prinzipien der Erfassung und Ordnung der Primärliteratur}

Aufnahme finden folgende Publikationsarten und -formen:

- die zu Nietzsches Lebzeiten erschienenen Drucke seiner Werke

- die posthumen Drucke seiner Werke, Werksammlungen, Briefe und Briefwechsel sowie der nachgelassenen Fragmente

- Drucke in Blindenschrift, Blindenhörbücher

- die Drucke seiner musikalischen Werke (Noten)

- Tonträger mit gesprochenen Werken und Einspielungen seiner musikalischen Werke

- Rezensionen zu allen Formen der Primärliteratur

Ausgeschlossen sind: Drucke einzelner Werke, Werkauszüge und Gedichte in Anthologien, anderen allgemeinen Textsammlungen, in Schulbüchern sowie in Zeitungen und Wochenblättern.

Was die "Vollständigkeit» bibliographischer Unternehmen dieser Art betrifft, so besteht sie nur als regulative Idee. Bei einzelnen Sprachbereichen wie z. B. Chinesisch, Griechisch, Koreanisch, Niederländisch und Russisch hat der Kontakt zu Nietzsche-Forschern oder zu Institutionen in den betreffenden Ländern viele wertvolle Literaturhinweise

${ }^{6}$ Besonders zu diesem Aspekt vgl. Frank Simon-Ritz: Ein personalbibliographisches Großprojekt - die Weimarer Nietzsche-Bibliographie. In: Informationsmittel für Bibliotheken (IFB) 5 (1997), H. 3/4, S. 735-740. 
erbracht. Besonders hervorzuheben ist die Hilfe, die wir aus Japan erhielten und die uns in die Lage versetzte, die japanischen Übersetzungen in hoher Vollständigkeit nachzuweisen.

Grundsätzlich wurde für die Erstellung der Titelaufnahmen Autopsie angestrebt. Allerdings waren v.a. Übersetzungen weder durch Leihverkehr noch durch Bibliotheksbesuche zu beschaffen. In diesen Fällen und auch beim Nachweis unveränderter Auflagen wurden Titel auch ohne Autopsie verzeichnet, sofern eine zuverlässige Quelle wie eine Nationalbibliographie die Existenz der Publikationen verbürgen konnte. Die Titelaufnahmen ohne Autopsie sind durch Sternchen (*) gekennzeichnet. Diese Kennzeichnung entfällt allerdings bei den chinesischen, japanischen und koreanischen Übersetzungen, die sämtlich ohne Autopsie aufgenommen wurden. Das gleiche gilt für die Rezensionen, die nicht durchweg autoptisch aufgenommen werden konnten.

Die Titelaufnahmen werden gemäß der ISBD (International Standard Bibliographic Description) und in Anlehnung an die RAK-WB (Regeln für die alphabetische Katalogisierung an wissenschaftlichen Bibliotheken) ausgeführt. Die Ansetzung der Personennamen und die Transliterierung aus den slawischen Sprachen und aus dem Griechischen erfolgt ebenfalls nach RAK-WB. Für das Chinesische richten wir uns nach Pinyin, für das Koreanische nach McCune/Reischauer, wobei jedoch vereinzelt auch Titel, welche nur in anderen Transkriptionen nachweisbar waren, Berücksichtigung fanden.

Die Bibliographie selbst ist formal-systematisch aufgebaut (vgl. dazu die "Systematische Gliederung «). Innerhalb der jeweiligen Systematikgruppen wird weiter geordnet nach die Systematik vertiefenden Zwischenüberschriften und Marginalien. Schließlich wird chronologisch aufsteigend nach Erscheinungsjahr, Erscheinungsort und Titel geordnet.

Die Titelaufnahmen werden in vielen Fällen durch Inhaltsangaben und durch Annotationen ergänzt. Die Inhaltsangaben erschließen v.a. die Werksammlungen in die Tiefe. Die Ansetzung der Titel in den Inhaltsangaben und auch im Werkregister geht auf die der maßgeblichen Editionen (BAW und $\mathrm{KGW}$ ) zurück, wo eine solche nicht erkennbar ist, wird sie [in eckiger Klammer] ergänzt. In den Annotationen wird vor allem auf editionsgeschichtliche Zusammenhänge hingewiesen.

Erschlossen wird die Bibliographie durch drei Register: Personenregister, Sachregister, Werkregister. Im Personenregister werden alle beteiligten Personen wie Herausgeber, Mitarbeiter, Übersetzer, Illustratoren, Rezensenten sowie die Korrespondenten Nietzsches verzeichnet. Das Sachregister enthält eine Reihe von formalen Schlagwörtern wie beispielsweise »Bibliophile Ausgaben«. Im Personenregister wie auch im Werkregister sind zudem eine Reihe von Verweisungen von abweichenden Namen- oder Titelformen auf die Ansetzungsformen enthalten.

\section{Dank}

Für die Ermittlung der relevanten Literatur sind die üblichen Wege beschritten worden. In hohem Maße wurden die Möglichkeiten des 
Internets für die Erarbeitung der Bibliographie genutzt. So konnten beispielsweise die Kataloge der wichtigsten Bibliotheksverbünde und Bibliotheken der Welt durchgesehen werden, bevor zur intensiven Auswertung und Autopsie vor Ort gezielt einzelne Bibliotheken besucht wurden. $\mathrm{Zu}$ den persönlich aufgesuchten Bibliotheken zählen: Staatsbibliothek zu Berlin - Preußischer Kulturbesitz (Auskunftsteam um Herrn Ziegler), die Bibliothek des Iberoamerikanischen Instituts - PreuBischer Kulturbesitz, Die Deutsche Bibliothek / Deutsche Bücherei Leipzig (Auskunftsteam um Frau Bertram), British Library London, Rossijskaja Gosudarstvennaja Biblioteka Moskva (Bibliografičeskij otdel), Vserossijskaja Gosudarstvennaja Biblioteka Inostrannoj Literatury Moskva, Bibliothèque Nationale Paris, Library of Congress Washington D.C. (Reference Crew um Joe Puccio). Allen Kolleginnen und Kollegen an diesen Institutionen, die uns zum Teil außerordentlich kooperativ zur Seite gestanden haben, sei herzlich gedankt.

Eine erhebliche Anzahl hier nicht einzeln aufzuführender Bibliotheken des In- und Auslandes und deren Mitarbeiter haben unsere Arbeit durch ihren unermüdlichen Einsatz in der Fernleihe und mit zahlreichen Auskünften unterstützt.

$\mathrm{Zu}$ danken haben wir einer Reihe von freundlichen Helfern und Korrespondenten, die uns in sehr großzügiger Weise bei bibliographischen Recherchen unterstützten und uns wichtiges Material für die Erfassung der Übersetzungen von Nietzsches Werken lieferten. Hier sei besonders Prof. Maki Shimizu (Hiroshima/Japan) für seine aufwendige Zuarbeit der japanischen Übersetzungen gedankt. Ohne seinen Beitrag wäre die Bibliographie in einem wichtigen Teilbereich unvollständig geblieben. Ebenso herzlich ist Prof. Franc Cengle (Belgrad/Jugoslawien), Dr. Pieter Mostert (Voorburg/Niederlande), Dr. Chiu-yee Cheung (Brisbane/ Australien), Natalija I. Lopatina (Moskva/Rußland) und Dr. Ernani Chaves (Belem/Brasilien) für ihre Hilfe zu danken.

Technische Unterstützung erhielten wir bei der Transliteration und Übersetzung der griechischen Titel von Pela Kalokyri, bei den türkischen Titeln von Yesim Kirac (beide Erfurt), bei den koreanischen Titeln von Helene Kang (Hamburg).

Finanzielle Förderung erfuhr das Projekt außer durch die DFG durch die Allianz AG / Zweigniederlassung Leipzig, die uns die Anschaffung eines Laptops für die Bibliotheksreisen ermöglichte. Insbesondere bedanken wir uns bei Dr. Günter Ullrich.

Die anspruchsvollen EDV-technischen Anforderungen wurden durch das EDV-Dezernat der Stiftung Weimarer Klassik und durch die Firma S\&T Systemtechnik Ilmenau erfüllt.

Für das Gelingen der »Weimarer Nietzsche-Bibliographie« keinesfalls weniger wichtig, bleibt unseren zeitweiligen studentischen Hilfskräften Corinna Deibel, Ines Böttcher, Ralf Dressel, Jan Lüdecke und Todor Simeonow ebenso zu danken wie dem gesamten Kollegium der Herzogin Anna Amalia Bibliothek.

Michael Knoche

Weimar, im Frühjahr 2000 


\section{Verzeichnis der verwendeten Abkürzungen}

Abkürzungen, die in der Nietzsche-Philologie üblich sind:

BAB

BAW

GA

GAK

Gast'sche Ausgabe

$\mathrm{GBr}$

GSA

HAAB

KA

KGB

KGW

Krummel I / II / III R. F. Krummel: Nietzsche und der deutsche Geist.

- Berlin ; New York. - Bd. 1-3. 1974-1998.

KSA

KSB

Mette, Nachlaß

MusA

Nachlaß

NWZ

SA

TA

Thatcher

WNB

Historisch-kritische Gesamtausgabe, Briefe (Bd.

1-4), vgl. Nr. 10

Historisch-kritische Gesamtausgabe, Werke (Bd.

1-5), vgl. Nr. 10

Großoktavausgabe (Bd. I-XIX), vgl. Nr. 3

Großoktavausgabe, soweit von Koegel ediert, vgl.

Nr. 2

Nietzsches Werke (abgebrochene Ausgabe 1893/4), vgl. Nr. 1

Gesammelte Briefe (Bd. I-V), vgl. Nr. 400

Goethe- und Schiller-Archiv Weimar

Herzogin Anna Amalia Bibliothek Weimar

Kleinoktavausgabe (Bd. I-XVI), vgl. Nr. 4

Kritische Gesamtausgabe Briefwechsel (Abt.I/

1-Abt.IV/4), vgl. Nr. 401

Kritische Gesamtausgabe Werke (Abt.I/1-Abt.

VIII/4), vgl. Nr. 14

Kritische Studienausgabe Sämtliche Werke (Bd.

1-15), vgl. Nr. 16

Kritische Studienausgabe Sämtliche Briefe (Bd.

1-8), vgl. Nr. 402

H. J. Mette: Der handschriftliche Nachlaß Friedrich Nietzsches. - Leipzig, 1932.

Musarionausgabe (Bd. I-XXIII), vgl. Nr. 7

Nietzsches Nachlaß (generell), heute im Goetheund Schiller-Archiv Weimar

Podach, Nietzsches Werke des Zusammenbruchs, vgl. Nr. 45

Werke, Bd. 1-3 u. Index-Bd., hrsg. von Schlechta, vgl. Nr. 12

Taschenausgabe (Bd. 1-11), vgl. Nr. 5

Thatcher, David S.: Musical settings of Nietzsche texts. - In: Nietzsche-Studien. ([T. 1]. - Bd. 4. 1975.

S. 284-323. - [T. 2]. - Bd. 5. 1976. S. 355-383. -

[T. 3]. - Bd. 15. 1986. S. 440-452)

Weimarer Nietzsche-Bibliographie 


\section{Allgemeine Abkürzungen:}

Wie die im folgenden Verzeichnis enthaltenen Wörter werden auch grammatikalisch unterschiedliche Formen dieser Wörter sowie stammverwandte Wörter der gleichen Sprache und anderer Sprachen abgekürzt.

\begin{tabular}{|c|c|c|c|}
\hline$(*)$ & $\begin{array}{l}\text { Titel ohne Autopsie } \\
\text { aufgenommen }\end{array}$ & $\begin{array}{l}\text { corr. } \\
\text { cur. }\end{array}$ & $\begin{array}{l}\text { corrected } \\
\text { curavit }\end{array}$ \\
\hline \multirow[t]{3}{*}[***]{} & Titel von Werken & d. & deel \\
\hline & $\begin{array}{l}\text { Nietzsches mit grie- } \\
\text { chischen Textbestand- }\end{array}$ & $\begin{array}{l}\text { Darst. } \\
\text { dass. }\end{array}$ & $\begin{array}{l}\text { Darstellung } \\
\text { dasselbe }\end{array}$ \\
\hline & $\begin{array}{l}\text { teilen, hier translite- } \\
\text { riert wiedergegeben }\end{array}$ & $\begin{array}{l}\text { Dept. } \\
\text { ders. }\end{array}$ & $\begin{array}{l}\text { Department } \\
\text { derselbe }\end{array}$ \\
\hline Abt. & Abteilung & d.h. & das heißt \\
\hline Akad. & Akademie & dies. & dieselbe \\
\hline Anh. & Anhang & dir. & direzione \\
\hline Anm. & Anmerkung & Diss. & Dissertation \\
\hline annot. & annotavit & dop. & dopolnenyj \\
\hline arg. & argang & Dr. & Druck \\
\hline Aufl. & Auflage & durchges. & durchgesehen \\
\hline Auftr. & Auftrag & ed. & edited \\
\hline augm. & augmenté & Einf. & Einführung \\
\hline Ausg. & Ausgabe & eingel. & eingeleitet \\
\hline ausgew. & ausgewählt & Einl. & Einleitung \\
\hline Ausw. & Auswahl & enl. & enlarged \\
\hline Ausz. & Auszug & enth. & enthält \\
\hline autoris. & autorisiert & erarb. & erarbeitet \\
\hline $\mathrm{Bd}$. & Band & Erg. & Ergänzung \\
\hline Bearb. & Bearbeiter & Erl. & Erläuterung \\
\hline bed. verm. & bedeutend vermehrt & ersch. & erschienen \\
\hline begr. & begründet & Erstausg. & Erstausgabe \\
\hline $\begin{array}{l}\text { Delger. } \\
\text { Beih. }\end{array}$ & $\begin{array}{l}\text { beigefugt } \\
\text { Beiheft }\end{array}$ & $\begin{array}{l}\text { erw. } \\
\text { évf. }\end{array}$ & $\begin{array}{l}\text { erweitert } \\
\text { évfolyam }\end{array}$ \\
\hline Beil. & Beilage & Ex. & Exemplar \\
\hline Beitr. & Beitrag & fac. & faculté \\
\hline betr. & betreffend & facs. & facsimile \\
\hline Bibliogr. & Bibliographie & Fak. & Fakultät \\
\hline Bl. & Blatt & Faks. & Faksimile \\
\hline bzw. & beziehungsweise & farb. & farbig \\
\hline с̌. & čast' & fasc. & fasciculus \\
\hline ca. & circa & Fass. & Fassung \\
\hline cah. & cahier & ford. & forditotta \\
\hline $\mathrm{CD}$ & Compact-Disc & Forts. & Fortsetzung \\
\hline cev. & ceviren & Fotogr. & Fotografie \\
\hline coll. & collection & g. & god \\
\hline collab. & collaboratore & geb. & gebunden \\
\hline comm. & comment & gedr. & gedruckt \\
\hline comp. & compiled & gegr. & gegründet \\
\hline compl. & completed & gef. & gefaltet \\
\hline
\end{tabular}




\begin{tabular}{|c|c|c|c|}
\hline geh. & geheftet & över- & övers. \\
\hline Geleitw. & Geleitwort & sättning & \\
\hline ges. & gesammelt & opl. & oplag \\
\hline Ges. & Gesellschaft & oprac. & opracowal \\
\hline $\begin{array}{l}\text { getr. Zäh- } \\
\text { lung }\end{array}$ & getrennte Zählung & $\begin{array}{l}\text { Orig. } \\
\text { otd. }\end{array}$ & $\begin{array}{l}\text { Original } \\
\text { otdel }\end{array}$ \\
\hline gez. & gezählt & otv. red. & otvestvennyj redaktor \\
\hline H. & Heft & overs. & oversettelse \\
\hline Habil.-Schr. & Habilitationsschrift & per. & perevod \\
\hline Hrsg. & Herausgeber & pererabot. & pererabotannyj \\
\hline Hs. & Handschrift & pl. & plate \\
\hline Ill. & Illustration & poslesl. & posleslovie \\
\hline impr. & imprimerie & Portr. & Porträt \\
\hline introd. & introduction & Ppbk. & Paperback \\
\hline ispr. & ispravlennyj & pr. & print \\
\hline izd. & izdanie & predg. & predgovor \\
\hline jegyz. & jegyzet & predisl. & predislovie \\
\hline Jg. & Jahrgang & pref. & preface \\
\hline $\mathrm{k}$. & kiadás & překl. & překlad \\
\hline Kap. & Kapitel & přel. & prèelozil \\
\hline $\mathrm{Kl}$. & Klasse & pres. & presentazione \\
\hline $\mathrm{kn}$. & kniga & prev. & prevod \\
\hline Komm. & Kommission & pril. & priloženie \\
\hline komment. & kommentiert & primeč. & primečanie \\
\hline Kt. & Karte & pról. & prólogo \\
\hline Lfg. & Lieferung & przekł. & przekład \\
\hline libr. & librairie & przezł. & przełozył \\
\hline $\begin{array}{l}\text { Literatur- } \\
\text { verz. }\end{array}$ & Literaturverzeichnis & $\begin{array}{l}\text { Pseud. } \\
\text { pt. }\end{array}$ & $\begin{array}{l}\text { Pseudonym } \\
\text { part }\end{array}$ \\
\hline Ltg. & Leitung & Publ. & Publikation \\
\hline maschinen- & maschinenschriftlich & Red. & Redakteur \\
\hline schriftl. & & Repr. & Reprint \\
\hline Mitarb. & Mitarbeiter & Reprod. & Reproduktion \\
\hline Mitw. & Mitwirkung & rev. & revidiert \\
\hline Ms. & Manuskript & Rez. & Rezension \\
\hline Nachdr. & Nachdruck & rist. & ristampe \\
\hline Nachf. & Nachfolger & roč. & ročník \\
\hline Nachtr. & Nachtrag & rocz. & rocznik \\
\hline Nachw. & Nachwort & S. & Seite \\
\hline nem. & nemeckij & s. & siehe \\
\hline Neudr. & Neudruck & s.a. & siehe auch \\
\hline N.F. & Neue Folge & Schallpl. & Schallplatte \\
\hline No. & Numéro & Ser. & Serie \\
\hline Notenbeisp. & Notenbeispiele & s. 1. & sine loco \\
\hline nouv. & nouveau & s. n. & sine nomine \\
\hline N.R. & Neue Reihe & sogen. & sogenannt \\
\hline Nr. & Nummer & sost. & sostavitel' \\
\hline N.S. & Neue Serie, new series & Sp. & Spalte \\
\hline & & Suppl. & Supplement \\
\hline
\end{tabular}




$\begin{array}{llll}\text { sz. } & \text { szám } & \text { veränd. } & \text { verändert } \\ \text { T. } & \text { Teil } & \text { veranst. } & \text { veranstaltet } \\ \text { t. } & \text { tome } & \text { verantw. } & \text { verantwortlich } \\ \text { Tab. } & \text { Tabelle } & \text { verb. } & \text { verbessert } \\ \text { Taf. } & \text { Tafel } & \text { verm. } & \text { vermehrt } \\ \text { teilw. } & \text { teilweise } & \text { vers. } & \text { versehen } \\ \text { tłum. } & \text { tłumaczył } & \text { vervielf. } & \text { vervielfältigt } \\ \text { Toncass. } & \text { Toncassette } & \text { Verz. } & \text { Verzeichnis } \\ \text { trad. } & \text { traduction } & \text { vgl. } & \text { vergleiche } \\ \text { transl. } & \text { translated } & \text { vgl. a. } & \text { vergleiche auch } \\ \text { Tsd. } & \text { Tausend } & \text { vol. } & \text { volume } \\ \text { u.a. } & \text { und andere, } & \text { vollst. } & \text { vollständig } \\ & \text { unter anderem } & \text { Vorabdr. } & \text { Vorabdruck } \\ \text { udg. } & \text { udgave } & \text { Vorbem. } & \text { Vorbemerkung } \\ \text { u.d.T. } & \text { unter dem Titel } & \text { vorm. } & \text { vormalig, vormals } \\ \text { überarb. } & \text { überarbeitet } & \text { Vorw. } & \text { Vorwort } \\ \text { Übers. } & \text { Übersetzer } & \text { vstupit. } & \text { vstupitel'nyj } \\ \text { übertr. } & \text { übertragen } & \text { vved. } & \text { vvedenie } \\ \text { überw. } & \text { überwiegend } & \text { vyd. } & \text { vydání } \\ \text { uitg. } & \text { uitgegeven } & \text { vyp. } & \text { vypusk } \\ \text { umgearb. } & \text { umgearbeitet } & \text { Wiss. } & \text { Wissenschaft } \\ \text { Univ. } & \text { Universität } & \text { wyd. } & \text { wydanie } \\ \text { unveränd. } & \text { unverändert } & \text { wydaw. } & \text { wydawnictwo } \\ \text { u.ö. } & \text { und öfter } & \text { zahlr. } & \text { zahlreich } \\ \text { uppl. } & \text { upplaga } & \text { zesz. } & \text { zeszyt } \\ \text { utg. } & \text { utgivare } & \text { z.T. } & \text { zum Teil } \\ \text { v.a. } & \text { vor allem } & \text { zugl. } & \text { zugleich } \\ \text { vál. } & \text { válgatott } & & \\ & & & \end{array}$




\section{Systematik der »Weimarer Nietzsche-Bibliographie«, Band 1: Primärliteratur}

$1.1 \quad$ Werksammlungen . . . . . . . . . . . . 3

1.1.1 Gesamtausgaben und größere Auswahlausgaben . . . . . 3

1.1.2 Teilsammlungen . . . . . . . . . . . . . . . 52

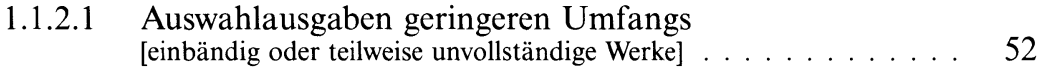

1.1.2.2 Sammlungen von Gedichten . . . . . . . . . . . . . 75

1.1.2.3 Sammlungen von nachgelassenen Fragmenten. »Der

Wille zur Macht«"

[Marginalie »Der Wille zur Macht « für die Kompilation des

Nietzsche-Archivs] . . . . . . . . . . . . . . . . . . . . . . . . . . . . . 90

1.1.3 Sammlungen von Werkauszügen . . . . . . . . . . . . . 94

$1.2 \quad$ Einzelschriften . . . . . . . . . . . . . . . . . . . . . . . . 104

1.2.1 Einzelne Werke . . . . . . . . . . . . . . . . . . . . 104

1.2.2 Nietzsche als Herausgeber . . . . . . . . . . . . . 151

1.3 Musikalisches Werk . . . . . . . . . . . . . . . . . 151

1.3.1 Sammlungen . . . . . . . . . . . . . . . 151

1.3.2 Einzelne Kompositionen . . . . . . . . . . . . . . 153

$1.4 \quad$ Briefe, Briefwechsel . . . . . . . . . . . . . . . 155

1.4.1 Gesamtausgaben und größere Sammlungen . . . . . . 155

1.4.2 Teilsammlungen (an mehrere Empfänger) . . . . . . . 158

1.4.3 Briefe an einzelne Empfänger, einzelne Briefwechsel . . . 160

1.5 Werke auf Datenträgern und Tonträgern

(Disketten, CD-ROMs, Schallplatten, Tonbänder, Compact-Discs) $\ldots 165$

1.5.1 Werksammlungen auf Datenträgern . . . . . . . . . . 165

1.5.2 Auszüge aus dem Werk auf Tonträgern . . . . . . . . 166

1.5.3 Werksammlungen und einzelne Werke auf Tonträgern . . 167

1.5.4 Einspielungen musikalischer Werke auf Tonträgern . . . 173

1.6 Übersetzungen

[jeweils durch Marginalien untergliedert: Ausgaben und Textsammlungen; - / Nachgelassene Fragmente; - / Der Wille zur Macht; Gedichte; Sammlungen von Auszügen; Einzelne Werke (in alphabetischer Folge);

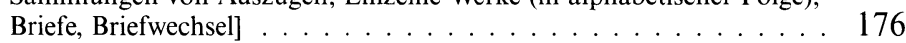

Albanisch . . . . . . . . . . . . . . . . 176

Arabisch . . . . . . . . . . . . . . . 177

Armenisch . . . . . . . . . . . . . . . . 177

Baskisch . . . . . . . . . . . . . . . 177

Belorussisch . . . . . . . . . . . . . . . 178

Bengali . . . . . . . . . . . . . . . 178

Bulgarisch . . . . . . . . . . . . . . 178

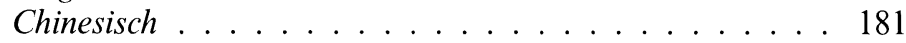

Dänisch . . . . . . . . . . . . . . . 192

Englisch . . . . . . . . . . . . . 195 
Systematische Gliederung der »Weimarer Nietzsche-Bibliographie»

Esperanto ................. 233

Estnisch . . . . . . . . . . . . 233

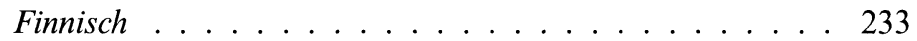

Französisch ..................... 235

Georgisch (Grusinisch) . . . . . . . . . . . . 281

Griechisch . . . . . . . . . . . . 281

Hebräisch . . . . . . . . . . . . . 285

Isländisch . . . . . . . . . . . . . . . . . . . 287

Italienisch . . . . . . . . . . . . . . . . . . 287

Japanisch . . . . . . . . . . . . . . . . . . . . . . . . . . . . . . . 329

Jiddisch . . . . . . . . . . . . . . . . . . . . . . 353

Katalanisch . . . . . . . . . . . . . . 354

Koreanisch . . . . . . . . . . . . . . . 355

Lettisch . . . . . . . . . . . . . . . . . . 364

Litauisch . . . . . . . . . . . . . . . . 365

Marathi ................. 365

Mazedonisch . . . . . . . . . . . . 366

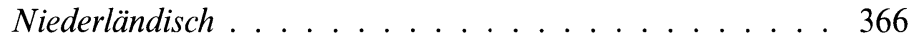

Norwegisch . . . . . . . . . . . . . 373

Persisch ....................... 375

Polnisch ................. . 376

Portugiesisch . . . . . . . . . . . . . . 382

Rumänisch . . . . . . . . . . . . . . . 395

Russisch . . . . . . . . . . . . . . . 399

Schwedisch . . . . . . . . . . . . . 415

Serbokroatisch ............... . . 419

Slowakisch . . . . . . . . . . . . . . . 426

Slowenisch . . . . . . . . . . . . . . . . . . 426

Spanisch . . . . . . . . . . . . . . . 428

Tschechisch . . . . . . . . . . . 467

Türkisch . . . . . . . . . . . . . . . . . . . . . . . . . . . . . . . 472

Ukrainisch . . . . . . . . . . . . . . 475

Ungarisch . . . . . . . . . . . . . . 475

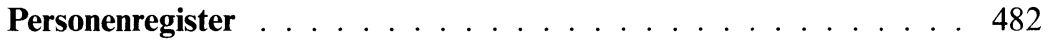

Sachregister ..................... 501

Werkregister .................... 502 